\title{
VLT/NACO adaptive optics imaging of the Herbig Ae star HD 100453`
}

\author{
X. P. Chen ${ }^{1}$, T. Henning ${ }^{1}$, R. van Boekel ${ }^{1}$, and C. A. Grady ${ }^{2}$ \\ 1 Max-Planck-Institut für Astronomie, Königstuhl 17, 69117 Heidelberg, Germany \\ e-mail: [chen; henning; boekel] @mpia.de \\ 2 Eureka Scientific and Goddard Space Flight Center, Exploration of the Universe Division, Code 667, NASA's GSFC Greenbelt, \\ MD 20771, USA \\ e-mail: cgrady@echelle.gsfc.nasa.gov
}

Revised 2 May 2005 / Accepted 4 October 2005

\section{ABSTRACT}

We report the detection of a companion candidate to the Herbig Ae star HD 100453, using the NACO instrument at the VLT UT4. The separation between the companion candidate and HD 100453 is 1".06, and their brightness contrast is $5.1 \mathrm{mag}$ at $K_{\mathrm{s}}$ and $4.2 \mathrm{mag}$ at Br $\gamma$. We discuss the possible nature of the newly detected star based on near-infrared photometry. The photometric data are consistent with a $0.3 M_{\odot}$ companion to HD 100453, corresponding to a spectral type of M3-M5. This detection could have important implications for the structure of the disk around HD 100453.

Key words. binaries: general - stars: individual: HD 100453 - stars: pre-main sequence - techniques: high angular resolution

\section{Introduction}

HD 100453 (IRAS 11307-5402, SAO 239162, HIP 56354) is an isolated Herbig Ae star of spectral type A9Ve (Malfait et al. 1998). It was not listed in the Herbig Ae/Be (HAEBE) catalogue of Thé et al. (1994), and was classified as a planetary nebula by García-Lario et al. (1997). However, recent observations suggest a Herbig nature of this star (Meeus et al. 2001, 2002; Vieira et al. 2003). Hipparcos measurements give a parallax of $8.97 \pm 0.76$ mas for HD 100453, from which a distance of $114_{-9}^{+11} \mathrm{pc}$ is derived. The position of the star in the HR diagram is close to the ZAMS and its age is estimated to be $\geq 10$ Myr (Meeus et al. 2002). Taking HD 100453 as one of the members of the Lower Centaurus Crux association (Kouwenhoven et al. 2005), the upper limit for the age of HD 100453 is 20 Myr (Mamajek et al. 2002). The luminosity of HD 100453 is relatively low $\left(L \sim 9 L_{\odot}\right)$ and its mass is estimated to be $1.7 M_{\odot}$ (Dominik et al. 2003).

The strong infrared excess in the spectral energy distribution (SED) of HD 100453 implies the presence of circumstellar dust which is believed to be located in a disk (Meeus et al. 2001, 2002; Dominik et al. 2003). According to the classification of Meeus et al. (2001) HD 100453 is a group Ib source, i.e., it has an SED that can be fitted with a power-law plus a black-body continuum, but does not show silicate emission bands shortward of $30 \mu \mathrm{m}$. On the other hand, moderately

\footnotetext{
* Based on data from the ESO/ST-ECF Science Archive Facility.
}

strong PAH bands are present at 3.3, 6.2, 7.7 and $11.2 \mu \mathrm{m}$ in the mid-IR spectrum of HD 100453 (Meeus et al. 2001). Both Meeus et al. (2002) and Dominik et al. (2003) suggested that the lack of a silicate feature is due to predominating large grains in the circumstellar disk of HD 100453. This viewpoint seems to be supported by the ISO-SWS spectrum of HD 100453. As shown in Vandenbussche et al. (2004), there is a tentative detection of an emission band in the $34 \mu \mathrm{m}$ region, which can be attributed to crystalline forsterite grains with a size of $\sim 2 \mu \mathrm{m}$.

In this paper we present VLT/NACO adaptive optics (AO) imaging of HD 100453 and the first detection of a faint source in its immediate vicinity. In Sect. 2 we describe the observations and present the results in Sect. 3. We discuss the possible effects of the detected faint object to the disk around HD 100453 in Sect. 4 and summarize the results in Sect. 5.

\section{Observations and data reduction}

The data were obtained from the ESO/ST-ECF Science Archive Facility $^{1}$. High-resolution images of HD 100453 (RA = $11^{\mathrm{h}} 33^{\mathrm{m}} 05.58^{\mathrm{s}}$, Dec $\left.=-54^{\circ} 19^{\prime} 28^{\prime \prime} .5(\mathrm{~J} 2000)\right)$ were taken on June 2nd 2003 with NACO (Lenzen et al. 2003; Lagrange et al. 2003). NACO is composed of NAOS, the first Adaptive Optics System on the VLT (Rousset et al. 2003) and CONICA, a $1-5 \mu \mathrm{m}$ imaging, coronographic, spectroscopic and polarimetric instrument (Lenzen et al. 1998).

\footnotetext{
${ }^{1}$ http://archive.eso.org
} 
Table 1. NACO observation logs of HD 100453 and No.9144. Camera S27 provides 27.0 mas/pixel sampling. DIT and NDIT correspond to the exposure integration time and the number of exposures, respectively.

\begin{tabular}{|c|c|c|c|c|c|c|c|}
\hline Source & Obs. date & $\begin{array}{c}\text { Central wavelength } \\
\qquad(\mu \mathrm{m})\end{array}$ & $\begin{array}{c}\text { Bandwidth } \\
(\mu \mathrm{m})\end{array}$ & $\begin{array}{l}\text { DIT } \\
(\mathrm{s})\end{array}$ & NDIT & $\begin{array}{l}\text { Seeing } \\
\left({ }^{\prime \prime}\right)\end{array}$ & airmass \\
\hline HD 100453 & June 02,03 & 2.18 & 0.35 & 2.0 & 2 & 0.9 & 1.153 \\
\hline HD 100453 & June 02,03 & 2.166 & 0.023 & 0.35 & 10 & 0.9 & 1.153 \\
\hline No.9144 & June 01,03 & 2.18 & 0.35 & 0.5 & 5 & 0.9 & 1.064 \\
\hline
\end{tabular}

The observations of HD 100453 were carried out in the $K_{\mathrm{s}}$ band $(2.18 \mu \mathrm{m})$ and the $\operatorname{Br} \gamma(2.166 \mu \mathrm{m})$ narrow band. The integration times are summarized in Table 1. The CONICA detector is an Aladdin InSb $1024 \times 1024$ pixel array. The camera used was S27 which provides a pixel scale of 0.0270 arcsec pixel ${ }^{-1}$ and a field of view of about $28^{\prime \prime} \times 28^{\prime \prime}$. During the observations, the mean visual seeing at the zenith was estimated to be 0.'9. Star No. 9144 in Persson et al. (1998; $\mathrm{RA}=10^{\mathrm{h}} 47^{\mathrm{m}} 24.1^{\mathrm{s}}$, Dec $\left.=-44^{\circ} 34^{\prime} 05^{\prime \prime}(\mathrm{J} 2000)\right)$ was selected from the ESO Archive to calibrate the photometry. It was observed on June 1st 2003 with a seeing of $0{ }^{\prime} 9$ and at an airmass of 1.064. The details of the observations are summarized in Table 1.

The data reduction was carried out using self-developed IDL scripts. After bad-pixel filtering, flat-field correction, and sky removal, the individual dithering positions were combined into a resulting mosaic image.

\section{Results}

Figure 1 presents the images of HD 100453 at $K_{\mathrm{s}}$ and $\operatorname{Br} \gamma$. The main result of the observations is the detection of a previously unknown source to the southeast of HD 100453. In the following we refer to this unknown source as HD 100453B, and refer to HD 100453 itself as HD 100453A. With respect to HD 100453A, we derive from the images a position angle of $127^{\circ} .3 \pm 1^{\circ}$ and a separation of $1^{\prime \prime} .06 \pm 0.02$ (projected linear separation $=120 \pm 2 \mathrm{AU}$ at the distance of $114 \mathrm{pc}$ ) for HD 100453B. The positional error given here is dominated by the centering uncertainties of HD 100453B (around 0.5 pixels).

Aperture photometry of HD 100453B and the calibration star No.9144 ( $K_{\mathrm{s}}=11.28 \mathrm{mag}$, Persson et al. 1998) was performed with the IRAF/APPHOT package. The photometric apertures of HD 100453B and the calibrator were set to 10 pixels $(0.27, \simeq 2.8 F W H M)$, in which most of the flux of HD 100453B was recovered. Accurate photometry of HD 100453B suffers from the additional background flux caused by HD 100453A. In order to reduce this effect, we integrated the background flux in the annulus between 49 pixels and 29 pixels with HD 100453A at the center (excluding the flux from HD 100453B), and derived the flux scale in this annulus, assuming circularly symmetric emission from HD 100453A. Then we calculated the background flux in the photometric aperture of HD 100453B and subtracted this background flux from HD 100453B. On the basis of the aperture photometry and error estimates, we derived a magnitude of $10.7 \pm 0.1 \mathrm{mag}$ for HD $100453 \mathrm{~B}$ in the $K_{\mathrm{s}}$ band.
Therefore, the brightness contrast between HD 100453A and HD $100453 \mathrm{~B}$ is $5.1 \mathrm{mag}$ in the $K_{\mathrm{s}}$ band (the magnitude of HD 100453A is $5.60 \pm 0.021 \mathrm{mag}$, which is taken from the 2MASS Point Source Catalog 2 ). Due to the high brightness contrast between primary and secondary, HD 100453B was below the detection limit of the near-infrared survey of Kouwenhoven et al. (2005) by roughly one magnitude, and thus remained undetected by these authors. The brightness contrast at $\mathrm{Br} \gamma$ is measured to be $4.2 \mathrm{mag}$ which is somewhat smaller than that at $K_{\mathrm{s}}$. The photometric apertures of HD 100453A and $\mathrm{B}$ at $\mathrm{Br} \gamma$ were also set to 10 pixels, close to the PSF FWHM of both sources. We also subtracted the background contribution from the flux of HD 100453B at $\operatorname{Br} \gamma$.

The proximity of HD 100453B to HD 100453A suggests that HD $100453 \mathrm{~B}$ is a physical companion to HD 100453A. HD 100453 is an isolated Herbig Ae star. The 2MASS survey indicates that the HD 100453 region is not densely populated. In a region with a radius of $5^{\prime}$ centered on HD $100453 \mathrm{~A}$, there are only 7 stars brighter than HD $100453 \mathrm{~B}\left(K_{\mathrm{s}}=10.66 \mathrm{mag}\right)$ in the $K$ band (see Cutri et al. 2003). In analogy to earlier binary surveys, we would consider any bright star closer than 2 .'0 to the primary as a companion. Therefore, the probability for a bright field star to be considered as a companion by chance is less than $\left[7 /(5 \times 60)^{2} \pi\right] \times 2.0^{2} \pi=3.0 \times 10^{-4}$. On the other hand, it is known that the binary frequency among HAEBE stars is very high, approximately $40 \%$ (see Bouvier \& Corporon 2001). This supports the view that HD $100453 \mathrm{~B}$ is a physical companion to HD $100453 \mathrm{~A}$.

The spectra of HD 100453 may provide further evidence for the existence of a companion. The $\mathrm{H} \alpha$ spectrum of HD 100453 is double peaked and shows an additional redshifted emission component (Meeus et al. 2002). It is possible that this additional redshifted emission actually comes from HD 100453B. Furthermore, the FUSE spectrum of HD 100453 also shows a sharp component superposed on a broad and complex double emission profile (Grady et al. in preparation). However, before we can state that the newly discovered object is really bound to HD 100453A, the binary hypothesis has to be checked by taking spectra of the companion candidate, which would need to show a very cool atmosphere, and by second epoch images to verify that the proper motions are identical.

Although we cannot definitely determine the nature of HD 100453B only from fluxes in the $K_{\mathrm{s}}$ band and the $\mathrm{Br} \gamma$ band, some tentative results can be drawn from its photometric properties. Hereafter a companion orbiting

\footnotetext{
2 http://vizier.cfa.harvard.edu/viz-bin/ VizieR?-source=II/246
} 

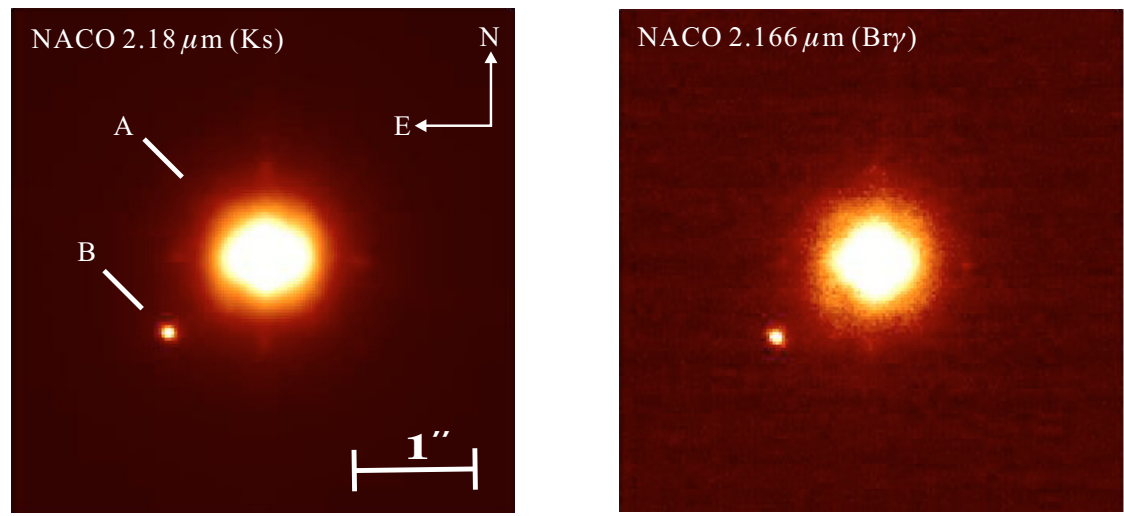

Fig. 1. VLT/NACO AO imaging of HD 100453 in the $K_{\mathrm{s}}$ band $(2.18 \mu \mathrm{m})$ and the $\mathrm{Br} \gamma$ band $(2.166 \mu \mathrm{m})$. North is up, and east is to the left. The positions of HD 100453A and HD 100453B are indicated with letters A and B. The scale is marked in the $K_{\mathrm{s}}$ band image.

HD 100453A at $\sim 120$ AU distance is considered. We assume that HD 100453A and HD 100453B have the same age, which is consistent with the result that the components in PMS binary systems are coevally formed (see Brandner \& Zinnecker 1997; White \& Ghez 2001). Then we take the distance and age of HD 100453B as $114 \mathrm{pc}$ and 10-20 Myr, respectively. A simple comparison to the evolutionary tracks of Baraffe et al. (1998) shows that the $K_{\mathrm{s}}$ magnitude of HD 100453B is in agreement with the predicted magnitudes of a star of 0.25 to $0.35 M_{\odot}$. The corresponding spectral types are M3-M5. An estimate of the orbital motion of a binary can be made once the linear separation of the components and the total mass have been specified. If we assume a circular orbit in the plane of the sky and assume that the masses of HD 100453A and HD 100453B are $1.7 M_{\odot}$ and $0.3 M_{\odot}$, the linear separation $(120 \pm 2 \mathrm{AU})$ yields an orbital period of $\sim 930 \mathrm{yr}$.

\section{Discussion}

The absence of the $10 \mu \mathrm{m}$ silicate emission feature in the spectrum of HD 100453 has been a puzzle (Meeus et al. 2002; Dominik et al. 2003; Vandenbussche et al. 2004). Since small amorphous silicate grains are the most abundant dust species in interstellar space, their spectral signature is expected to be present during all stages of the star formation process. However, in ISO spectra, Meeus et al. (2001) found four objects which do not show silicate emission (including HD 100453) in their sample of 14 HAEBE stars. Acke \& van den Ancker (2004) also reported the non-detection of silicate emission for 16 out of their sample of 46 objects. These observations imply that the absence of the silicate emission feature is a relatively common phenomenon among HAEBE stars. Since the emission feature is due to small warm silicate grains, the absence of the feature must mean that such grains are not anymore present. Where have these grains gone?

Based on the passive disk models by Dullemond et al. (2001), disk geometry effects were once considered (see the discussion in Meeus et al. 2002). However, the models required an unusually large scale-height of the inner rim to efficiently suppress the $10 \mu \mathrm{m}$ silicate emission (see Dominik et al. 2003). Recently it was also shown that the shadowing of the inner rim is not efficient enough to suppress the $10 \mu \mathrm{m}$ silicate emission (Dullemond \& Dominik 2004). Meeus et al. (2002) suggested two other possible causes for the absence of the $10 \mu \mathrm{m}$ silicate feature: (1) the small grains are removed by radiation pressure and/or Poynting-Robertson (P-R) drag; (2) the grains in the disk have experienced more coagulation than those in other systems with a similar energy distribution, leading to a strong depletion of small grains. Since there are some HAEBE stars of a similar age to HD 100453 and with higher luminosities (e.g. HD 100546, $L \sim 32 L_{\odot}$ ) that also still show a lot of small warm silicate grains, in general the second explanation is more favored (see Meeus et al. 2002; Dominik et al. 2003; Vandenbussche et al. 2004).

If the absence of the $10 \mu \mathrm{m}$ silicate emission feature in HD 100453 is really due to the dust growth in the disk, the question turns to why HD 100453 and similar sources have experienced more efficient coagulation than other sources with comparable characteristics (e.g., ages, luminosities, and SEDs). What is the unique feature of these sources leading to the absence of the $10 \mu \mathrm{m}$ silicate emission? The detection of the companion to HD 100453 may provide a clue to this problem. A number of physical mechanisms might affect the evolution of circumstellar material in a binary system. On the one hand, when a companion is surrounding the primary, the viscous effect of the companion will intensify the perturbation of the material close to the primary, which will increase the dynamical activity of the grains in the inner disk. At the same time, when the companion is clearing the disk and forming a gap (see below), the grain density in the rim of the inner disk will increase simultaneously due to the sweeping-up by the companion. Both the increase of the activity and the density should accelerate the coagulation of the grains in the inner disk (see the review by Beckwith et al. 2000), which may explain why HD 100453 can coagulate small warm grains more efficiently than sources with similar properties.

On the other hand, in close binary systems, circumstellar disks can be tidally truncated and this truncation can clear the material in the disk (see the review by Lubow \& Artymowicz 2000). Some indirect studies of disk clearing have been made through an SED analysis (see Jensen \& Mathieu 1997). The essential idea is that cleared regions remove emitting material of a 
Table 2. The sample of HAEBE sources without $10 \mu \mathrm{m}$ silicate feature.

\begin{tabular}{cccccc}
\hline \hline Source & Group & Binary & $\begin{array}{c}\text { Angular } \\
\text { Separation } \\
\text { (arcsec) }\end{array}$ & $\begin{array}{c}\text { Linear } \\
\text { Separation } \\
\text { (AU) }\end{array}$ & References \\
\hline HD 34282 & I & $?$ & - & - & - \\
HD 34700 & I & yes & $6 \times 10^{-4}$ & 0.26 & Torres (2004) \\
HD 100453 & I & yes & 1.06 & 120 & This work \\
HD 135344 & I & $?$ & - & - & - \\
HD 169142 & I & yes & 7.7 & 939 & Grady et al. in preparation \\
BD+40 4124 & I & $?$ & - & - & - \\
MWC1080 & I & yes & 0.76 & 1220 & Leinert et al. (1997) \\
HD 35187 & II & yes & 1.3 & 195 & Maheswar et al. (2002) \\
RR Tau & II & $?$ & - & - & - \\
HD 141569 & II & yes & 7.55 & 760 & Weinberger et al. (2000) \\
Wray 15-1484 & II & $?$ & - & - & - \\
LkH $\alpha 224$ & II & $?$ & - & - & - \\
\hline
\end{tabular}

characteristic temperature, thereby leading to a deficit of emission at corresponding wavelengths. Jensen \& Mathieu (1997) studied the SEDs of some young spectroscopic binaries with disks in order to search for disk clearing. They indeed found that some of the binaries had no near-infrared excess emission, but substantial mid- and far-infrared excesses, indicating the signature of cleared inner disks. The inferred sizes of these inner cleared holes are consistent with sizes of the binary orbits. Theoretical research suggests that the exact range of stable orbits depends on the binary mass ratio and orbital eccentricity, and these cleared regions of the disk are approximately $0.5 a$ for circular orbits or $0.2 a$ for eccentric orbits (where $a$ is the binary separation; Artymowicz \& Lubow 1994). It is generally accepted that the $10 \mu \mathrm{m}$ silicate emission originates in the inner region of the disk. According to the theoretical prediction, the outer radius of the disk around HD 100453A should be less than $60 \mathrm{AU}$ or even $20 \mathrm{AU}$, if the linear distance of $120 \mathrm{AU}$ is representative for the semi-major axis of the system. Although at the present we do not know to what extent silicate grains can be cleared by the companion at this distance (a few tens of AU), the effect of clearing by the companion cannot be excluded at this point. Such clearing should reduce the abundance of small silicate grains in the disk and lead to the observed weakness of the silicate emission feature. On-going high-spatial resolution observations (e.g., MIDI data) will provide constraints on the location of the grains emitting in the infrared, which is also helpful for further understanding the nature of the silicate emission in circumstellar disks, and to estimate to what extent small silicate grains can be affected by clearing due to close companions.

To investigate the effect of the companions on the $10 \mu \mathrm{m}$ silicate emission feature, we searched the literature for the binary characteristics of the four sources without a $10 \mu \mathrm{m}$ silicate emission compiled by Meeus et al. (2001). After excluding HD 139614 (which does show silicate emission, van Boekel et al. 2005), two out of the remaining three sources (HD 169142, Grady et al. 2005, in preparation;
HD 100453, this work), have companions. If we take the sources listed in Acke \& van den Ancker (2004) into account, there are 6 sources with established companions among the 12 sources lacking a silicate emission feature (see Table 2). It must be noted here, however, that out of these 6 systems, 3 are wide (of order $10^{3} \mathrm{AU}$ ) binaries. In these systems, the influence of the companion on the region of the disk responsible for the silicate emission, is likely negligible. Furthermore, as suggested by Acke \& van den Ancker (2004), the presence of the $10 \mu \mathrm{m}$ feature in some cases may be masked by the presence of strong PAH emission in the 8.6 and 11.2 micron bands. The binary characteristics of 6 sources in this sample are still unknown. Further investigation is needed to establish whether Herbig stars that lack a $10 \mu \mathrm{m}$ silicate feature show a higher binary frequency than stars with a silicate feature.

\section{Conclusion}

Based on ESO Archive data, we reported the detection of a companion to the Herbig Ae star HD 100453. The separation between the companion candidate and HD 100453 is $11^{\prime \prime} 06$, corresponding to a linear separation of 120 AU. The brightness contrast between the primary and companion candidate is $5.1 \mathrm{mag}$ at $K_{\mathrm{s}}$ and $4.2 \mathrm{mag}$ at $\mathrm{Br} \gamma$. We discussed the possible nature of the newly detected source based on near-infrared photometry. The $K_{\mathrm{s}}$ band magnitude of the source is consistent with a $0.3 M_{\odot}$ companion around HD 100453, corresponding to a spectral type of M3-M5. Assuming a circular orbit in the plane of the sky, the mass and linear separation yield an orbital period of $\sim 930 \mathrm{yr}$.

Acknowledgements. This research is based on observations made with the European Southern Observatory telescopes obtained from the ESO/ST-ECF Science Archive Facility. C. A. Grady was supported through HST-GO-9136 to Eureka Scientific. 


\section{References}

Acke, B., \& van den Ancker, M. E. 2004, A\&A, 426, 151

Artymowicz, P., \& Lubow, S. H. 1994, ApJ, 421, 651

Baraffe, I., Chabrier, G., Allard, F., \& Hauschildt, P. H. 1998, A\&A, 337, 403

Beckwith, S. V. W., Henning, T., \& Nakagawa, Y. 2000, in Protostars and Planets IV, ed. V. Mannings, A. P. Boss, \& S. R. Russell (Tucson: Univ. Arizona Press), 533

Bouvier, J., \& Corporon, P. 2001, in The Formation of Binary Stars, ed. H. Zinnecker, \& R. D. Mathieu (San Francisco: ASP), IAU Symp., 200, 155

Brandner, W., \& Zinnecker, H. 1997, A\&A, 321, 220

Cutri, R. M., et al. 2003, The 2MASS All-Sky Catalog of Point Sources

Dominik, C., Dullemond, C. P., Waters, L. B. F. M., \& Walch, S. 2003, A\&A, 398, 607

Dullemond, C. P., \& Dominik, C. 2004, A\&A, 417, 159

Dullemond, C. P., Dominik, C., \& Natta, A. 2001, ApJ, 560, 957

García-Lario, P., Manchado, A., Pych, W., \& Pottasch, S. R. 1997, A\&AS, 126, 479

Jensen, E. L., \& Mathieu, R. D. 1997, AJ, 114, 301

Kouwenhoven, M. B. N., Brown, A. G. A., \& Zinnecker, H. 2005, A\&A, 430, 137

Lagrange, A. M., Chauvin, G., Fusco, T., et al. 2003, SPIE, 4841, 860

Lenzen, R., Hofmann, R., Bizenberger, P., \& Tusche, A. 1998, SPIE, 3354,606
Lenzen, R., Hartung, M., Brandner, W., et al. 2003, SPIE, 4841, 944

Lubow, S. H., \& Artymowicz, P. 2000, in Protostars and Planets IV, ed. V. Mannings, A. P. Boss, \& S. R. Russell (Tucson: Univ. Arizona Press), 731

Leinert, Ch., Richichi, A., \& Haas, M. 1997, A\&A, 318, 472

Maheswar, G., Manoj, P., \& Bahtt, H. C. 2002, A\&A, 387, 1003

Malfait, K., Bogaert, E., \& Waelkens, C. 1998, A\&A, 331, 211

Mamajek, E. E., Meyer, M. R., \& Liebert, J. 2002, AJ, 124, 1670

Meeus, G., Waters, L. B. F. M., Bouwman, J., et al. 2001, A\&A, 365, 476

Meeus, G., Bouwman, J., Dominik, C., Waters, L. B. F. M., \& de Koter, A. 2002, A\&A, 392, 1039

Persson, S. E., Murphy, D. C., Krzeminski, W., Roth, M., \& Rieke, M. J. 1998, AJ, 116, 2475

Rousset, G., Lacombe, F., Puget, P., et al. 2003, SPIE, 4839, 140

Thé, P. S., de Winter, D., \& Pérez, M. R. 1994, A\&AS, 104, 315

Torres, G. 2004, AJ, 127, 1187

van Boekel, R., Min, M., Waters, L. B. F. M., et al. 2005, A\&A, in press [arXiv: astro-ph/0503507]

Vandenbussche, B., Dominik, C., Min, M., et al. 2004, A\&A, 427, 519

Vieira, S. L. A., Corradi, W. J. B., Alencar, S. H. P., et al. 2003, AJ, 126, 2971

Weinberger, A. J., Rich, R. M., Becklin, E. E., Zuckerman, B., \& Matthews, K. 2000, ApJ, 544, 937

White, R. J., \& Ghez, A. M. 2001, ApJ, 556, 265 